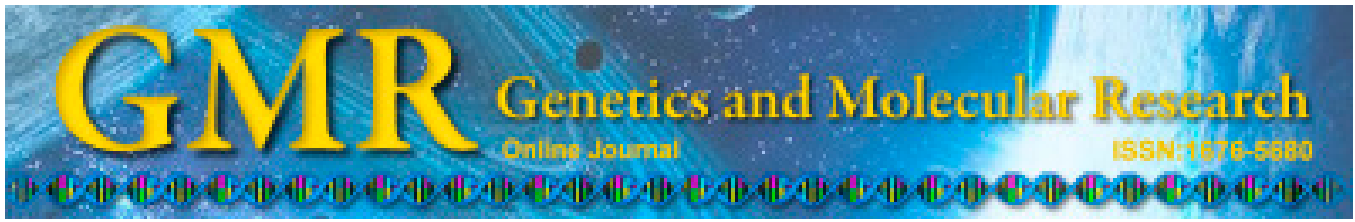

\title{
Proteomic analysis revealed the altered kidney protein profile of a Cyld knockout mouse model
}

\author{
Y. Zhao ${ }^{1,2 *}$, Y. Zhang ${ }^{1,2 *}$, H.B. Song ${ }^{1,2}$, F. Wu ${ }^{1,2}$, X.L. Wang ${ }^{2}$, S.-C. Sun ${ }^{3}$, \\ T.X. Cui ${ }^{2,4}$ and D.Q. Tang ${ }^{1,2}$ \\ ${ }^{1}$ Center for Stem Cell \& Regenerative Medicine, \\ The Second Hospital of Shandong University, Jinan, China \\ ${ }^{2}$ Shandong University Qilu Hospital Research Center for Cell Therapy, \\ Key Laboratory of Cardiovascular Remodeling and Function Research, \\ Qilu Hospital of Shandong University, Jinan, China \\ ${ }^{3}$ Department of Immunology, \\ The University of Texas MD Anderson Cancer Center, Houston, TX, USA \\ ${ }^{4}$ Department of Cell Biology and Anatomy, \\ University of South Carolina School of Medicine, SC, USA \\ *These authors contributed equally to this study. \\ Corresponding authors: D. Tang / T. Cui \\ E-mail: tangdq@sdu.edu.cn / taixing.cui@uscmed.sc.edu
}

Genet. Mol. Res. 14 (2): 5970-5978 (2015)

Received August 20, 2014

Accepted November 3, 2014

Published June 1, 2015

DOI http://dx.doi.org/10.4238/2015.June.1.14

\begin{abstract}
The aim of this study was to compare the proteomics pattern of the kidneys from Cyld knockout mice with that from normal mouse kidneys and establish a preliminary understanding of the role of Cyld in the kidney. Proteins from the kidneys of knockout Cyld mice and wild-type mice were extracted, isobaric tags for relative and absolute quantitation (iTRAQ) was performed, and the proteomics patterns of the two groups were compared. The genotypes of the mice were verified by polymerase chain reaction. A total of 1748 proteins with a local false discovery rate of $\leq 5 \%$ were identified, among which 1437 proteins were reliably recognized and quantified. The expression of two dysregulated proteins was confirmed by Western blotting. Gene
\end{abstract}


ontology and pathway analyses indicated that the proteins identified were involved in biological processes, cell components, and molecular functions, and participated in different pathways. Some of the proteins identified were relevant to renal function or kidney diseases. The difference between the proteomics profiles of kidneys from Cyld knockout mice and wild-type mice was prominent, which correlates to kidney dysfunction and the development of renal diseases.

Key words: iTRAQ; Cyld knockout; Kidney disease; Proteomics

\section{INTRODUCTION}

As an important system for protein degradation, the ubiquitin proteasome system (UPS) regulates numerous cellular biochemical processes via the ubiquitination of substrate proteins and the participation of the proteasome (Demartino and Gillette 2007; Mukhopadhyay and Riezman, 2007; Schulman and Harper, 2009). The UPS is a precisely regulated system in which deubiquitinating enzymes play a significant role.

$C Y L D$ was first identified as a suppressor gene of human familial cylindromatosis. The CYLD protein was later classified as a deubiquitinating enzyme, and is involved in multiple signal pathways (Massoumi, 2010). CYLD negatively regulates the NF- $\mathrm{KB}$ pathway, which plays a pathogenic role in mediating chronic inflammation in chronic kidney disease (CKD) (Rangan et al., 2009). The NF-kB pathway is consistently activated throughout the entire disease progression of anti-glomerular basement membrane (anti-GBM) glomerulonephritis, inducing various target genes, including complement 3 (C3), IL-Ib, IL-6, TRAF1, and SAA1. Moreover, George et al. (2012) recently discovered the role of NF-kB-p65 in antioxidant and redox homeostasis in renal cells by demonstrating that NF- $\mathrm{kB}-\mathrm{p} 65$ increases the expression of antioxidant enzymes (GPx1, SOD-1). Cui et al. (2009) found that CYLD plays a protective role in tubulointerstitial inflammation caused by IgA nephropathy, and Sun et al. (2009) reported similar results. A case reported by Ströbel et al. (2002) suggests that a mutation of $C Y L D$ is closely associated with the pathogenesis of spiradenocylindroma.

Proteomics combined with mass spectrometry (MS) has become a powerful paradigm for examining biological processes in a generalized manner by revealing unknown biological functions (Sui et al., 2008; Jin et al., 2009). In particular, isobaric tags for relative and absolute quantification (iTRAQ) labeling followed by multidimensional liquid chromatography-mass spectrometry/mass spectrometry (LC-MS/MS) analysis facilitates the detection of proteins in complex mixtures (Ross et al., 2004). To determine the specific effect of CYLD on the kidney and its mechanism of action, we performed proteomic analysis on kidney samples from Cyld knockout mice.

\section{MATERIAL AND METHODS}

\section{Cyld knockout mouse model}

The generation and verification of Cyld knockout mice has been described previously (Zhang et al., 2006). A section of the tail of each mouse was collected for polymerase chain reaction verification. 


\section{Protein extraction and quantification}

Proteins were extracted from the kidneys of Cyld knockout and wild-type (WT) mice $(\mathrm{N}=6$ per group). The kidneys in each group were pooled and homogenized in ice-cold phosphate-buffered saline containing a protease inhibitor cocktail (Roche Complete Mini Tablets, Roche Applied Sciences; Indianapolis, IN, USA). The supernatant contained the extracted proteins. The concentrations of the protein extracts were determined using the Bradford method (Bradford, 1976). The protein samples were stored at $-80^{\circ} \mathrm{C}$ for isoelectric focusing or iTRAQ analysis.

\section{iTRAQ labeling}

The iTRAQ labeling technique was used to compare the protein expression profiles of kidneys from Cyld knockout and WT mice. iTRAQ labeling enabled the comprehensive analysis of differential proteome expression, as described previously (Jin et al., $2009,2011)$. For iTRAQ LC-MS/MS analysis, $50 \mu \mathrm{g}$ clarified supernatants was denatured for $1 \mathrm{~h}$ at $60^{\circ} \mathrm{C}$, the disulfide bonds were reduced, and the cysteine residues were blocked, as described in the iTRAQ protocol (Applied Biosystems, Foster City, CA, USA). The two groups of kidneys, Cyld knockout and WT, were labeled using iTRAQ reagents 119 and 121 , respectively.

\section{Strong cation exchange chromatography}

The dried sample was resuspended in $100 \mu \mathrm{L}$ buffer $\mathrm{A}\left(10 \mathrm{mM} \mathrm{H}_{3} \mathrm{PO}_{4} / \mathrm{KH}_{2} \mathrm{PO}_{4}\right.$ in an aqueous solution of $25 \%$ acetonitrile and acidified to a $\mathrm{pH}$ of 3.0 using phosphoric acid). Each sample was then separated via off-line strong cation exchange chromatography using the Agilent 1200 HPLC system (Agilent, Palo Alto, CA, USA). The first fraction from 1-5 min was collected, and then each fraction was collected for 3-min intervals from 5-35 min; the final fraction from 35-46 min was collected, making a total of 12 fractions. All fractions were dried in a vacuum-freezing dryer for LC-MS/MS analysis.

\section{Reverse-phase LC-MS/MS analysis}

The samples were resuspended in re-dissolving solution, loaded on a C18RP precolumn $(100 \mu \mathrm{m} \times 3 \mathrm{~cm}, \mathrm{C} 18,3 \mu \mathrm{m}, 150 \AA)$, and desalted for $10 \mathrm{~min}$ using the Eksigent nanoLC-Ultra $^{\mathrm{TM}}$ 2D System (Eksigent Technologies, USA). Separation was performed using an elution gradient from 5 to $35 \%$ beginning with Eluant A (consisting of $94.9 \%$ deionized water, $5.0 \%$ methanol, and $0.1 \%$ formic acid, $\mathrm{pH}=3$ ) and transitioning to Eluant B (5.0\% deionized water, $94.9 \%$ methanol, and $0.1 \%$ formic acid) over $70 \mathrm{~min}$. The LC-MS/MS analysis was performed using a TripleTOF 5600 System (Applied Biosystems/ MDSSIEX), and the MS data were acquired in information-dependent acquisition mode. The data were processed using the mouse database of the Protein Pilot Software version 4.0 (AB SCIEX). The tolerances were specified as $\pm 0.05 \mathrm{Da}$ for peptides and $\pm 0.05 \mathrm{Da}$ for MS/ MS fragments. False discovery rate (FDR) analysis was also performed using the internal tools of ProteinPilot. Only the proteins identified with a local FDR $\leq 5 \%$ were considered for further analysis. 


\section{Bioinformatic analysis}

To improve the confidence of protein quantification, the iTRAQ expression ratios of the proteins were based on the criterion that the protein must be identified by a minimum of two peptides with $\geq 95 \%$ confidence. Proteins were considered to be differentially expressed if the iTRAQ ratios were $\geq 1.5$ or $\leq 0.67$ in the kidneys of the $C y l d$ knockout mice relative to the WT mice. Gene Ontology (GO) analysis was performed using the DAVID toolkit. Public resources, such as NCBI (The National Center for Biotechnology Information), PIR (Protein Information Resource), and GO, were used to integrate the protein ID and the corresponding gene ontology information. The proteins were classified into groups according to biological process (BP), molecular function (MF), and cell component (CC). Pathway enrichment was performed by comparing the annotated enzymes in the query dataset to the Kyoto Encyclopedia of Genes and Genomes (KEGG) database. Interactions between the query proteins were collected from the string database.

\section{Western blot analysis}

Dysregulated protein expression in Cyld knockout kidney samples was verified by Western blot analysis as described before (Masui et al., 2013). The proteins used (and their antibodies) were: Hsd17b4 (Abcam ab170910) and AKR1B3 (ABcam ab175394).

\section{RESULTS}

\section{Verification of the Cyld knockout model}

The genotypes of mice from groups 119 and 121 were verified to be Cyld knockout and WT, respectively (

\section{Identification and validation of differentially expressed proteins}

We identified 1748 proteins with a local FDR $\leq 5 \%$, among which 1437 proteins were reliably recognized and quantified (at least 2 peptides were identified) (Table S1). By measuring the ratio of 119:121, we determined that 20 proteins were upregulated and 20 proteins were downregulated in the Cyld knockout kidneys (Table 1). A large proportion of the proteins are relevant to renal function or kidney disease.

\section{Validation of dysregulated protein expression}

To validate our MS analysis, we confirmed the differential expressions of two dysregulated proteins by Western blot analysis using samples from the Cyld knockout and WT groups. The peroxisomal multifunctional enzyme (Hsd17b4) and aldose reductase (AKR1B3) were selected for validation based on their interesting biology and potential significance in kidney tumors (Figure 1). 
Table 1. Heat map of 30 selected proteins. This heat map displays the change in the expression level of selected dysregulated proteins. Fold change of proteins varies from 0.12 to 16.90 .

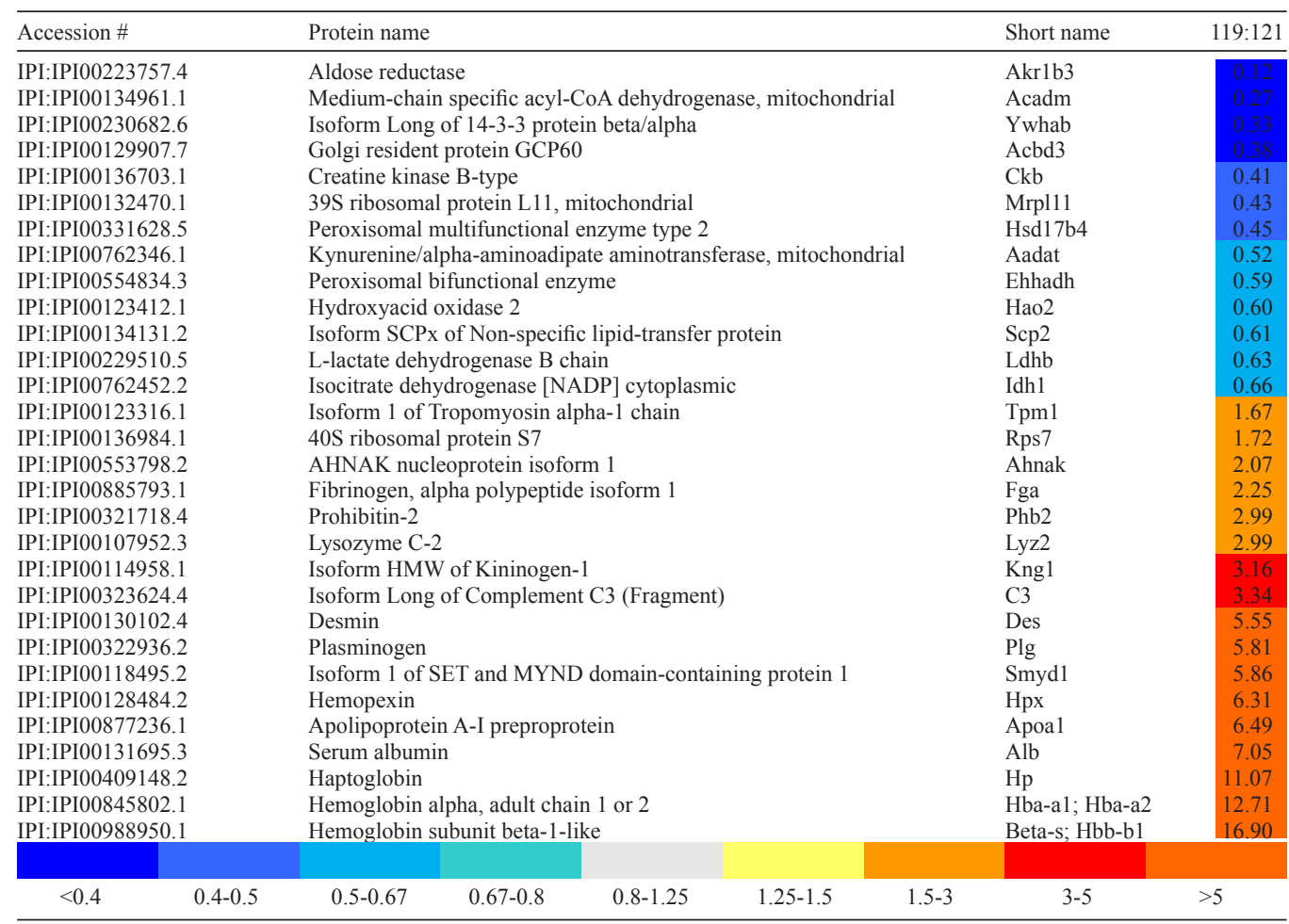

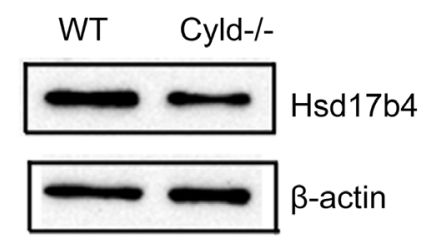

WT Cyld-/-
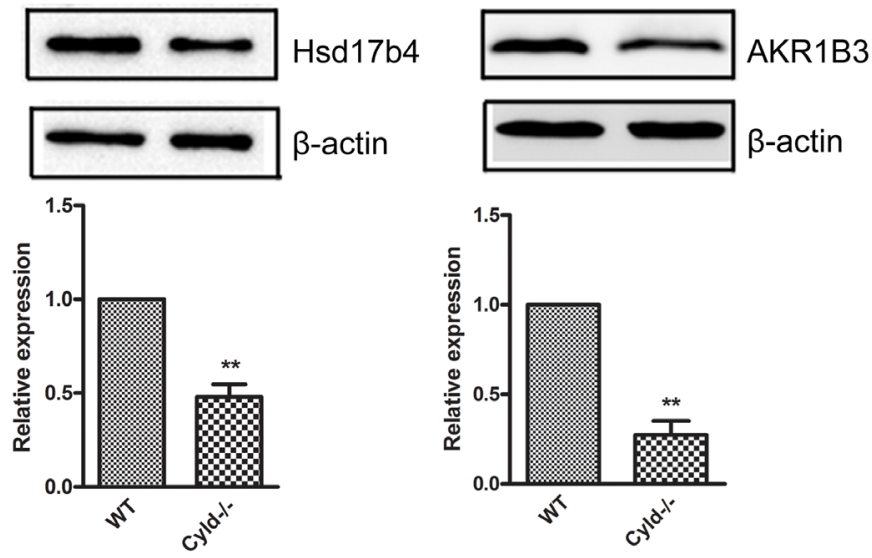

Figure 1. Verification of Hsd17b4 and AKR1B3 in Cyld knockout and WT by Western blot analysis. Representative blots showing the expression of Hsd17b4 and AKR1B3 in Cyld knockout and WT. $\beta$-actin was used as a loading control. The bar graphs represent the ratios of Hsd17b4/ $\beta$-actin and AKR1B3/ $\beta$-actin, and were normalized against the control group. Data are reported as means \pm standard error $(\mathrm{SE})$ of three independent experiments $(* * \mathrm{P}<0.01)$. 


\section{GO analysis}

We subjected the 40 differentially expressed proteins in Cyld knockout mice to GO analysis and three categorizations were performed: BP, CC, and MF. Based on the analyses, $36(92.3 \%), 34(87.2 \%)$, and $37(94.9 \%)$ proteins were classified according to $\mathrm{BP}, \mathrm{CC}$, and $\mathrm{MF}$, respectively.

For BP, 45 biological processes were enriched, 34 of which displayed a $\mathrm{P}$ value $<0.05$. The top 10 enriched processes are presented here (Figure 2A), all of which are involved in the energy metabolism process. For $\mathrm{CC}, 13$ different cellular components were enriched, 10 of which displayed a $\mathrm{P}$ value $<0.05$. The top 10 components are presented here (Figure 2B), which are located in the mitochondria and the sarcomeres. For MF, 14 different groups were enriched, 9 of which displayed a $P$ value $<0.05$, which are presented in Figure $2 \mathrm{C}$. These processes included coenzyme binding and cellular metabolism.

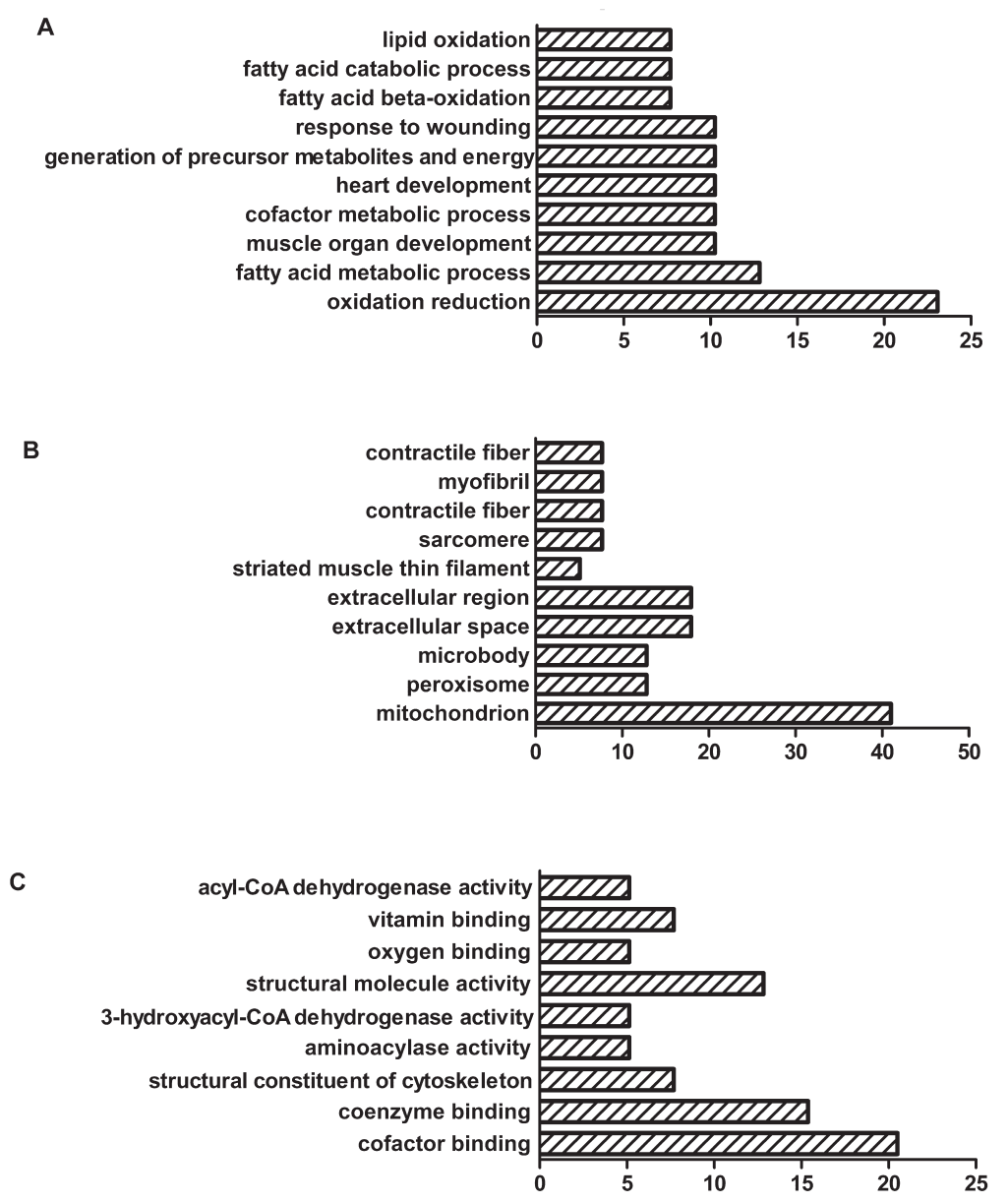

Figure 2. Gene Ontology (GO) analysis. Differentially expressed proteins for Cyld knockout group were tested for enrichment in GO terms of biological process (BP), cell component (CC), and molecular function (MF). For each category, the top 10 enriched processes are present here (A. BP, B. CC, and C. MF). For all groups, P $<0.05$. 


\section{Pathway analysis}

Pathway enrichment was performed by comparing the annotated enzymes in the query dataset to the KEGG database. Twenty-five proteins in total were mapped into 10 pathways, 7 of which displayed a $\mathrm{P}$ value $<0.05$. All of the enriched pathways containing more than 2 query proteins mapped are presented in Figure 3 . The primary related pathways were amino acid metabolism, the complement and coagulation cascades, and the peroxisome proliferatoractivated receptors (PPAR) signaling pathway.

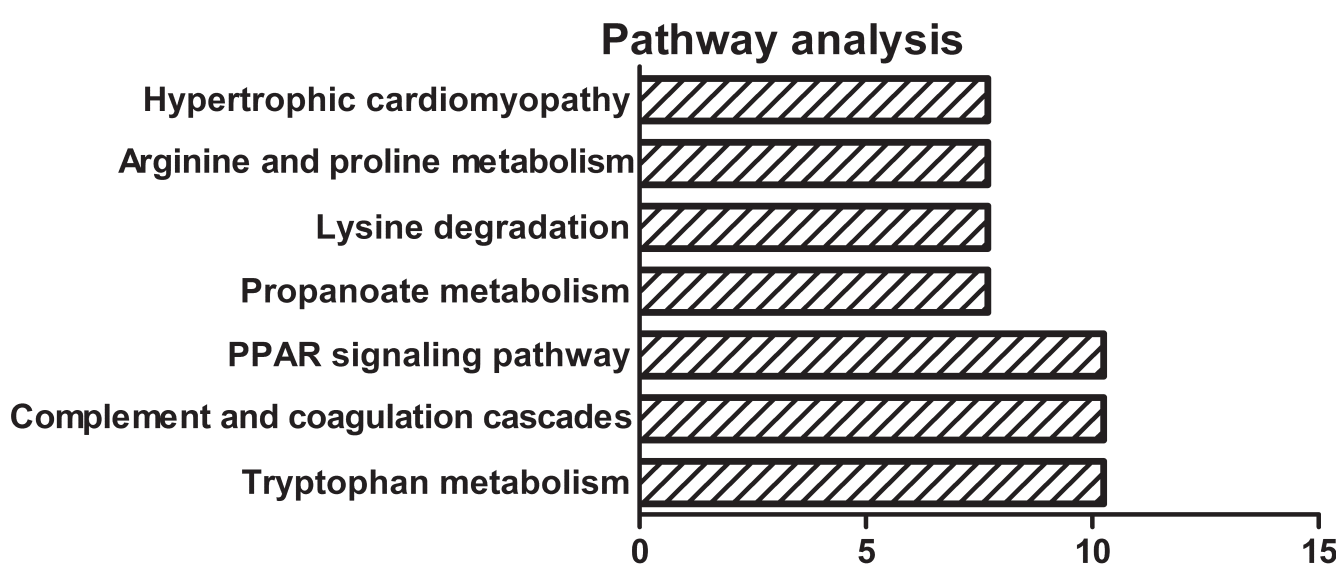

Figure 3. Pathways involving dysregulated proteins. The top 3 pathways in which the proteins were included were tryptophan metabolism, the complement and coagulation cascades, and the peroxisome proliferator-activated receptors (PPAR) signaling pathway.

\section{DISCUSSION}

Kidney diseases are life-threatening, non-communicable diseases that diminish a patient's quality of life. CKD is the general outcome of most types of kidney disease and has now become one of the most common causes of death. It is estimated that more than $10 \%$ or 20 million of individuals aged 20 years or older in the United States suffer from CKD and require dialysis treatment or a kidney transplant [data from the Centers for Disease Control and Prevention (CDC), USA].

To our knowledge, our study is the first to profile the proteome of kidneys from Cyld knockout mice using the iTRAQ technique. A total of 1437 proteins in total were reliably identified, which constituted a database of proteins for the Cyld knockout model. Among the dysregulated proteins, a large proportion are relevant to renal function or kidney disease.

AKR1B3, which is downregulated in adrenal tumors (Lefrançois-Martinez et al., 2004), is downregulated after Cyld knockout (fold change: 0.12), indicating that deletion of Cyld may increase the risk of adrenal tumor formation. It is also reported that the polymorphism of AKR1B1 (the human equivalent of AKR1B3) is closely associated with diabetic autonomic neuropathy (Donaghue et al., 2005), which suggests that AKR1B1 could be used as an early biomarker of autonomic neuropathy. Another report revealed that homozygotes of the aldose reductase $\mathrm{z}-2$ allele exhibited elevated plasma creatinine levels and an increased risk of classic diabetic glomerulopathy (Zhao et al., 2004), and we propose that the deletion of Cyld 
might help to reduce the risk of classic diabetic glomerulopathy. The expression of Hsd17b4, the peroxisomal multifunctional enzyme type 2 , is increased in esophageal cancer and prostate cancer (Li et al., 2005; Rasiah et al., 2009). It is also a biomarker of breast cancer (Flanagan et al., 2009). Considering the similarity between renal tumors and the cancers mentioned above, we predicted that Hsd17b4 is also upregulated in renal tumors. However, based on our results, Hsd17b4 is downregulated in the case of Cyld knockout mice (fold change: 0.45), implying that Cyld is a risk factor of renal tumor formation.

As shown in Figure 3,10.3\% of the dysregulated proteins are involved in the PPAR pathway. It has been well demonstrated that the PPAR $\gamma$ pathway is crucial for maintaining renal function, and PPAR agonists have been widely utilized to treat $\mathrm{CKD}$, progressive kidney disease, and diabetes (Yang et al., 2009; Fogo, 2011; Boor, 2012). On the other hand, PPAR $\gamma$ inhibits the expression of NF-kB induced by high glucose levels (Yang et al., 2009), which suggests that to some extent, both Cyld and PPAR $\gamma$ prevent the pathological progression from high glucose levels to diabetes. It is also possible that the PPAR $\gamma$ pathway is positively regulated by Cyld based on their equivalent ability to inhibit the expression of NF- $\mathrm{KB}$.

The complement and coagulation cascades are also important pathways in which $10.3 \%$ of the dysregulated proteins participate. Many components of these pathways are involved in the inflammation response and the loss of renal function (Yang et al., 2009). Several proteins in these pathways are upregulated; for example, PLG, KNG, FG, and $\mathrm{C} 3$ have foldchanges in expression of 5.81, 3.16, 2.25, and 3.34, respectively. These results imply that deletion of Cyld may enhance the inflammatory response. Therefore, Cyld may perform an anti-inflammatory role in renal tissue.

It is important to note that this experiment is predominantly based on proteomic analysis of the kidneys from Cyld knockout mice and should be regarded as an estimation of the proteomic profile, which provides potential biomarkers of kidney diseases. To further explore the function of individual proteins, renal function should be evaluated, and ultrasound should be performed to detect any morphological changes in the kidney.

\section{Conflicts of interest}

The authors declare no conflict of interest.

\section{ACKNOWLEDGMENTS}

Research supported by the National Qianren Scholar Program Special Funding of Shandong University and the Taishan Scholar Special Funding of Shandong Province.

\section{Supplementary material}

\section{REFERENCES}

Boor P (2012). New approaches in progressive kidney diseases. Pathologe. 33 (Suppl 2): 296-301.

Bradford MM (1976). A rapid and sensitive method for the quantitation of microgram quantities of protein utilizing the principle of protein-dye binding. Anal. Biochem. 72: 248-254.

Cui TG, Ichikawa T, Yang M, Dong X, et al. (2009). An emerging role of deubiquitinating enzyme cylindromatosis (CYLD) in the tubulointerstitial inflammation of IgA nephropathy. Biochem. Biophys. Res. Commun. 390: 307-312. Demartino GN and Gillette TG (2007). Proteasomes: machines for all reasons. Cell 129: 659-662. 
Donaghue KC, Margan SH, Chan AK, Holloway B, et al. (2005). The association of aldose reductase gene (AKR1B1) polymorphisms with diabetic neuropathy in adolescents. Diabet. Med. 22: 1315-1320.

Flanagan JM, Munoz-Alegre M, Henderson S, Tang T, et al. (2009). Gene-body hypermethylation of ATM in peripheral blood DNA of bilateral breast cancer patients. Hum. Mol. Genet. 18: 1332-1342.

Fogo AB (2011). PPARgamma and chronic kidney disease. Pediatr. Nephrol. 26: 347-351.

George LE, Lokhandwala MF and Asghar M (2012). Novel role of NF-kappaB-p65 in antioxidant homeostasis in human kidney-2 cells. Am. J. Physiol. Renal. Physiol. 302: F1440-1446.

Jin J, Park J, Kim K and Kang Y (2009). Detection of differential proteomes of human beta-cells during islet-like differentiation using iTRAQ labeling. J. Proteome Res. 8: 1393-1403.

Jin J, Kwon YW, Paek JS and Cho HJ (2011). Analysis of differential proteomes of induced pluripotent stem cells by protein-based reprogramming of fibroblasts. J. Proteome Res. 10: 977-989.

Lefrançois-Martinez AM, Bertherat J, Val P, Tournaire C, et al. (2004). Decreased expression of cyclic adenosine monophosphate-regulated aldose reductase (AKR1B1) is associated with malignancy in human sporadic adrenocortical tumors. J. Clin. Endocrinol. Metab. 89: 3010-3019.

Li XD, Liu XY, Huang XP, Fu JH, et al. (2005). Altered expression of the HSD17B4 gene in esophageal squamous cell carcinoma and loss of heterozygosity analysis. Zhongguo Yi Xue Ke Xue Yuan Xue Bao 27: 270-273.

Massoumi R (2010). Ubiquitin chain cleavage: CYLD at work. Trends Biochem. Sci. 35: 392-399.

Masui O, White NM, DeSouza LV, Krakovska, et al. (2013). Quantitative proteomic analysis in metastatic renal cell carcinoma reveals a unique set of proteins with potential prognostic significance. Mol. Cell. Proteomics 12: 132-144.

Mukhopadhyay D and Riezman H (2007). Proteasome-independent functions of ubiquitin in endocytosis and signaling. Science 315: 201-205.

Rangan G, Wang Y and Harris D (2009). NF-kappaB signalling in chronic kidney disease. Front Biosci (Landmark Ed) 14: 3496-3522.

Rasiah KK, Gardiner-Garden M, Padilla EJ, Möller G, et al. (2009). HSD17B4 overexpression, an independent biomarker of poor patient outcome in prostate cancer. Mol. Cell. Endocrinol. 301: 89-96.

Ross PL, Huang YN, Marchese JN, Williamson B, et al. (2004). Multiplexed protein quantitation in Saccharomyces cerevisiae using amine-reactive isobaric tagging reagents. Mol. Cell. Proteomics 3: 1154-1169.

Schulman BA and Harper JW (2009). Ubiquitin-like protein activation by E1 enzymes: the apex for downstream signalling pathways. Nat. Rev. Mol. Cell. Biol. 10: 319-331.

Ströbel P, Zettl A, Ren Z, Starostik P, et al. (2002). Spiradenocylindroma of the kidney: clinical and genetic findings suggesting a role of somatic mutation of the CYLD1 gene in the oncogenesis of an unusual renal neoplasm. Am. J. Surg. Pathol. 26: 119-124.

Sui J, Zhang J, Ching CB and Chen WN (2008). Comparative proteomic analysis of extracellular proteins reveals secretion of T-kininogen from vascular smooth muscle cells in response to incubation with s-enantiomer of propranolol. Mol. Pharm. 5: 885-890.

Sun F, Zheng X, E J, Liu G, et al. (2009). Tumor suppressor cylindromatosis: expressed in IgA nephropathy and negatively associated with renal tubulo-interstitial lesion. Chin. Med. J. (Engl). 122: 2603-2607.

Yang J, Zhang D, Li J, Zhang X, et al. (2009). Role of PPARgamma in renoprotection in Type 2 diabetes: molecular mechanisms and therapeutic potential. Clin. Sci. (Lond). 116: 17-26.

Zhang J, Stirling B, Temmerman ST, Ma CA, et al. (2006). Impaired regulation of NF-kappaB and increased susceptibility to colitis-associated tumorigenesis in CYLD-deficient mice. J. Clin. Invest. 116: 3042-3049.

Zhao HL, Tong PC, Lai FM, Tomlinson B, et al. (2004). Association of glomerulopathy with the 5'-end polymorphism of the aldose reductase gene and renal insufficiency in type 2 diabetic patients. Diabetes 53: 2984-2991. 